\title{
On Matters Arising
}

\author{
Constructive criticism is paramount to the advance of scholarly debates but comes with challenges.
}

$\mathrm{R}$ esearch articles are one of the most trusted ways for scholars to disseminate research advances within the academic community, and increasingly so beyond academia. The dissemination of research is essential to move scholarly debates forward and build up knowledge. Research findings are quite often used to inform government decisions about policies and interventions, especially in the case of sustainability challenges that have impacts on the well-being of people and the planet. The peer review of research articles is meant to ensure the quality and robustness of the research reported and aims at building trust in the value and usefulness of the findings discussed. Peer-reviewed articles are therefore one of the pillars of the academic research system and, rightly so, are subject to scrutiny. In a way, the peer review of an article doesn't really stop with journal publication. This is true generally, and especially so in the context of interdisciplinary sustainability research. It is not uncommon to witness the emergence of heated debates about published articles. Critiques cover data collection approaches, methodologies for analysis, framing of research questions, or even misconceptions about the ramifications of the issue under study. Pre-publication peer review is intended to control for all those possible fallacies and more. It should only let work that is solid, robust and reproducible get through to publication, and it does so for the most part, but it's far from perfect and, despite the best efforts of referees and journal editors, on occasions it can fail to identify some of those issues.

Scrutiny after publication is therefore as vital as scrutiny before publication. Sound and constructive critiques aimed at exposing and discussing potential important weaknesses in the design and/ or the execution of a study can lead to vital debates that, in turn, further understanding of topics of interest. As a matter of fact, scholarly debates, including the most intense ones, ultimately inspire new research. So, peer-reviewed journals clearly have a duty to make space for post-publication critiques - and such contributions need to be evaluated independently by experts in the same vein as original studies are. Yet,

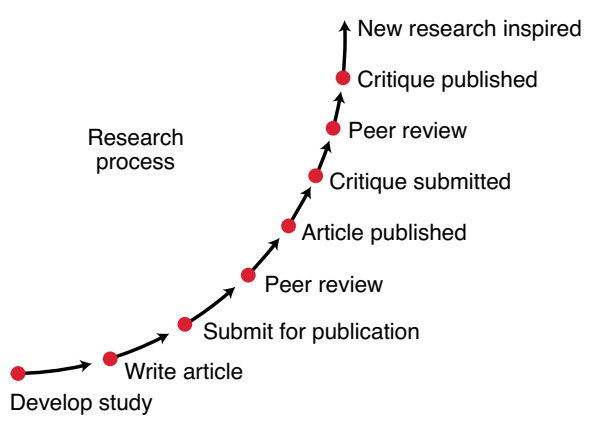

peer review of post-publication comments could suffer from the same limitations as the (pre-publication) peer review of the articles criticized. Besides, editorially assessing the relevance to the broad research community of these post-publication critiques is often challenging, particularly in the case of the typical multi-faceted research questions posed by sustainability scholars.

At Nature Sustainability, like all other Nature-branded journals, we offer the Matters Arising (https://www.nature.com/ natsustain/info/matters-arising) format for post-publication critiques to articles recently featured in the journal. Publishing Matters Arising contributions is critical to voice substantive concerns and foster debate. But it's not plain sailing. Practically, Matters Arising articles use up editorial and publishing capacity that would otherwise be in support of handling new research papers. Hence, editors must select the critiques that, by challenging the original article published, are most likely to advance the research discourse and that are most likely to engage a substantial portion of readers. Comments of philosophical nature, for example criticizing the research framing of a study from specific points of view, are not suitable for Matters Arising unless they challenge assumptions on the basis of scholarly arguments of relevance beyond a specific field or line of thinking. Likewise, critiques of specialist nature, for example related to aspects of a particular method of interest to a specific research field, are unlikely to pass editorial selectivity, unless they pose serious doubts on the robustness of the published findings. This doesn't mean that such critiques are not valuable, they most certainly are. But, like in the case of the many solid research articles editors reject without sending them to referees, we must also make difficult choices in the case of Matters Arising submissions. And it is worth reminding our readers that, as the journal evolves and matures, editorial selectivity adjusts both for original research and Matters Arising contributions.

More explicitly, here are some of the questions the editors ask when it comes to deciding whether or not to consider a Matters Arising comment for publication: is the critique substantive enough to undermine the conclusions of the study and would it matter to readers beyond a specific research field? Does it provide a useful and accessible account of the likely weaknesses? Does it suggest a constructive way forward? Was the criticized article recently published (within a few months from the Matters Arising submission)? If the editors answer yes to most of these questions, and provided the critique is not based on a misunderstanding of the published article, a response from the criticized authors is invited and the full exchange is then peer reviewed by a common set of referees. Referees are asked to evaluate the soundness and importance of the weaknesses highlighted and of the response offered. Editors finally decide on publication, balancing editorial considerations - the extent to which the exchange highlights important limitations that need consideration in future research and beyond a specific field - with the technical notes from independent reviewers.

Overall decisions to consider critiques to published articles are challenging, and particularly so in the case of a highly selective journal like Nature Sustainability. Although editors do value sound critiques generally, they need to choose those challenging published papers on substantive grounds, with broad implications to the relevant research issues. This is our ethos, and this is what makes Nature Sustainability a venue for the most valuable original research as well as the most useful critiques to advance the scholarly debates that will contribute to a brighter future.

Published online: 12 August 2020 https://doi.org/10.1038/s41893-020-00599-x 